\title{
Spontaneous coherent cyclotron THz super-radiation from a short dense photo-injector electron bunch
}

\author{
Yu.S. Oparina and A.V. Savilov
}

Institute of Applied Physics, N.Novgorod, Russia, savilov@appl.sci-nnov.ru

Laser-driven photo-injectors allow formation of compact and accessible sources of dense electron bunches with a moderate energy of 3-6 MeV, picosecond pulse durations, and charges of up to $1 \mathrm{nC}$ and even greater. These bunches can be used for realization of comparatively simple and compact terahertz sources operating in the regime of spontaneous coherent undulator radiation. This type of radiation is realized, when the effective phase size of the electron bunch with respect to the wave is small enough $(<2 \pi)$, so that the wave packets emitted by each of the electrons add up basically in phase. The $\mathrm{THz}$ source based on the spontaneous emission has a number of advantages as compared to the more traditional free-electron laser (FEL) based on the undulator emission induced due to bunching of a long electron beam by the radiated wave. These are a relatively high efficiency of the emission from the "ready-for-radiation" bunch, a narrow frequency band of the radiated rf signal in a system without a feedback system and an input rf signal, and the phase of the radiated rf signal fixed by the electron bunch phase. These advantages make the $\mathrm{THz}$ source based on the spontaneous emission attractive for a number of important applications, including the use of synchronization of such an oscillator with the X-ray FEL in pump-probe experiments [1-4]. The radiation, which could be characterize as induced radiation, take place, then effective phase size of bunch approximately $2 \pi$. In this case electron bunches are formed by spontaneous radiation of bunch edges. The $\mathrm{THz}$ source based on the spontaneous emission has a number of advantages. These are a relatively high efficiency of the emission from the "ready-for-radiation" bunch, a narrow frequency band of the radiated $\mathrm{rf}$ signal in a system without a feedback system and an input rf signal, and the phase of the radiated rf signal fixed by the electron bunch phase.
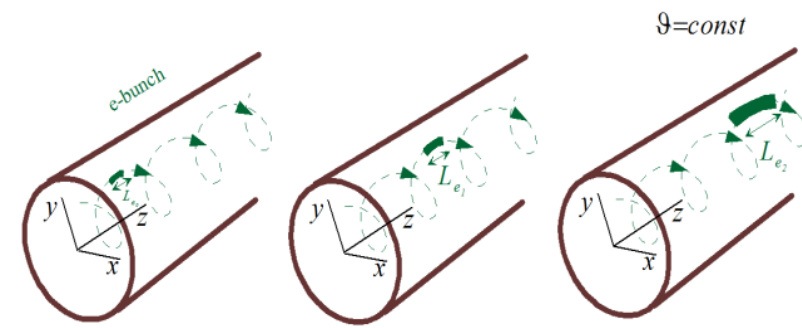

Fig. 1. The surface of electron bunch's identical phase and the evolution of the bunch length in the case of the group and cyclotron resonance regime

However, essential to the realization of a THz source based on the spontaneous coherent radiation is the presence of the mechanism of stabilization of the phase size of the bunch with respect to the radiated wave. Actually, the spontaneous emission occurs while the bunch phase size is smaller than $2 \pi$, so that wave packets emitted by each of the electrons add up basically in phase. In the case of the ubitron (as well as Cherenkov) source, this means that the bunch length must stay shorter than the radiation wavelength during the whole radiation region. Therefore, the length of the radiation region of such an $\mathrm{rf}$ source is strictly limited by the axial Coulomb repulsion, which is very strong in short dense photo-injector bunches and lead to a significant spreading of the bunch during its motion through a path being as short as few decimeters.
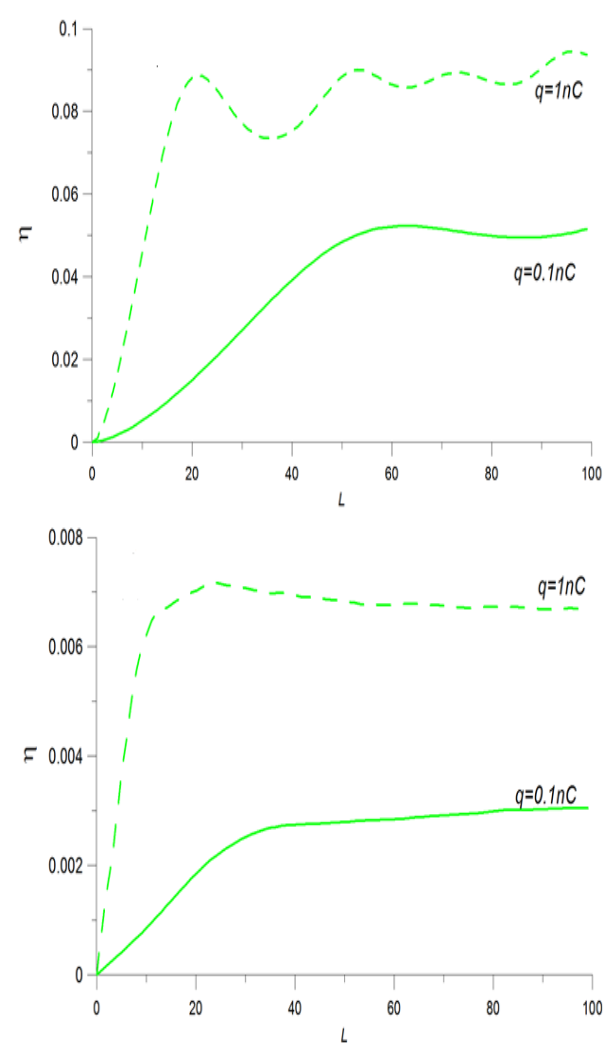

Fig. 2. Efficiency of the electron-wave interaction as a function of cavity length for two different charges of the electron bunch in the case of the group resonance (on the top) and then the group velocity of wave smaller than bunch's velocity (on the bottom)

In this work, we show that this problem is solved automatically in the case, when the cyclotron mechanism of the spontaneous coherent radiation is used. If the group resonance regime is used (the axial electron velocity is close to the group velocity of the radiated wave), then an increase in the length of the bunch (due to the Coulomb repulsion) does not lead (at least in the first approximation) to an increase in the phase size of the bunch (see fig. 1). The change of resonance phase $\vartheta=\omega_{0} t-h_{0} z-\int \Omega_{c} / \gamma d t$ (there $\Omega_{c}=e B_{0} / m_{e} c-$ the cyclotron frequency of electrons, $\omega_{0}$ - frequency of radiated 
wave, $\gamma$ - relativistic Lorentz-factor, $h_{0}-$ axial wave number), which is caused by coulomb repulsion, is determined by follow equation

$$
\frac{\partial \vartheta}{\partial\left(\omega_{0} t\right)} \approx-\left(1-\beta_{\| 0}{ }^{2}\right) \frac{\beta_{g r}}{\beta_{\| 0}} \frac{\Delta \gamma_{j c}}{\gamma_{0}}+\left(1-\beta_{\| 0} \beta_{g r}\right) b \frac{\Delta \gamma_{j c}}{\gamma_{0}}+\Delta,
$$

there $\beta_{0}=V_{\| 0} / c, V_{\| 0}-$ the initial axial velocity of the electron bunch, $\beta_{g r}=V_{g r} / c, V_{g r}$ - group wave's velocity, $b=B_{0} / B_{0 r e s}-$ the factor of the cyclotron mismatch, $\Delta=\left(1-\beta_{g r} \beta_{00}\right)(1-b)-$ the mismatch of the electronwave resonance. Obviously, the electron cyclotron phase with respect to the radiated wave includes both axial and azimuthal terms, and the changes in these two terms caused by a variation in electron energy due by the Coulomb repulsion have opposite signs, it follows of equation for the electron phase.
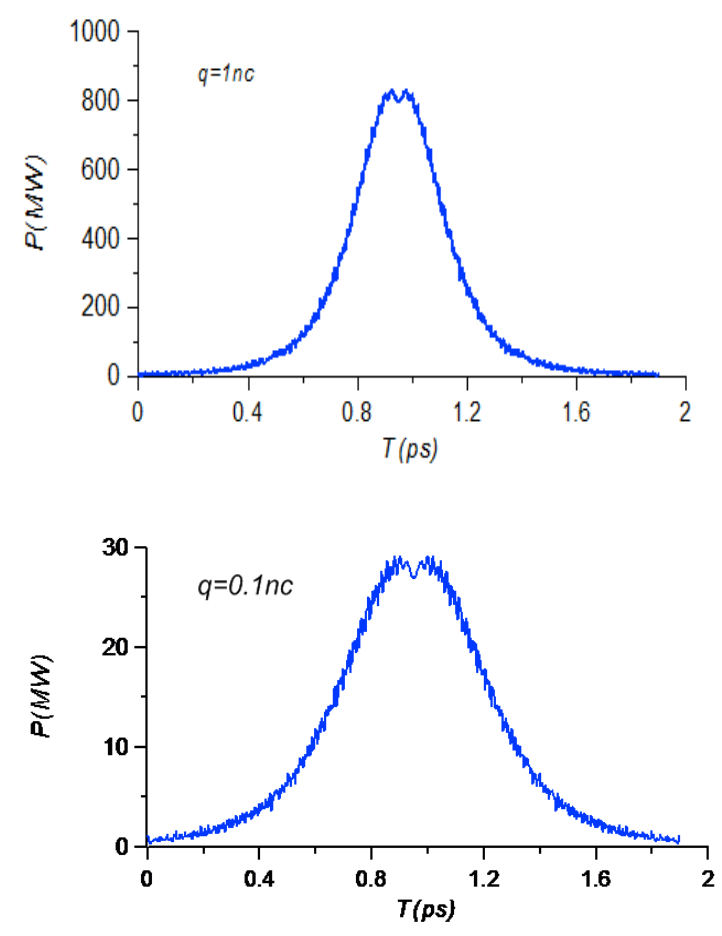

Fig. 3. Radiated pulses in the case of group resonance
The total mutual compensation takes place, when the group resonance regime is provided. It is important, that the group resonance regime is optimal also from the point of view of the maximization of the output $\mathrm{rf}$ power (see fig. 2), as in this regime the rf wave emission has the super-radiative character.

We have an opportunity to generate pulses with level of the power of tens-hundreds MW and duration of $1 \mathrm{pc}$, using spontaneous coherent radiation at the frequency $0.5 \mathrm{THz}$ from short bunches with charges $0.1-1 \mathrm{nc}$ (see fig. 3). Also it is possible to realize higher power with the spontaneous radiation of $\sim 10 \mathrm{nC}$ bunches (see fig.4).

The work is supported by IAP RAS Project 00352014-0012, and Russian Foundation for Basic Research Project 15-42-02260.

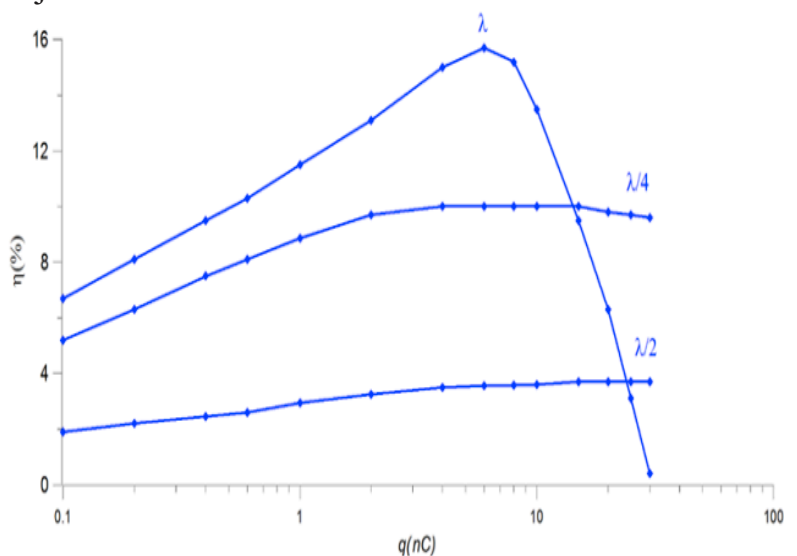

Fig. 4. Efficiency of the electron-wave interaction as a function of the bunch charge for three different bunch lengths

\section{References}

1. E. Chiadroni, A. Bacci, M. Bellaveglia, M. Castellano, G.Di. Pirro el al. J. of Physics: Conf. Series 359, 012018 (2012).

2. E.A. Schneidmiller, M.V. Yurkov, M. Krasilnikov, F. Stephan. Proceedings of SPIE 8778, 877811 (2013).

3. P. Boonpornprasert, M. Khojoyan, M. Krasilnikov, F. Stephan, B. Marchetti, E. Schneidmiller, M. Yurkov, S. Rimjaem. Proc. 36th International Free Electron Laser Conference FEL 2014 (Basel, Switzerland), Pages 153-158.

4. L. Wibmann, M. Emons, M. Kellert, K. Kruse, G. Palmer, M. Pergament, G. Priebe, J. Wang, U. Wegner, and M. J. Lederer. Proc. 36th International Free Electron Laser Conference FE. 